\title{
A New LMI Condition for Delay-Dependent Asymptotic Stability of Delayed Hopfield Neural Networks
}

\author{
Shengyuan Xu, James Lam, Senior Member, IEEE, and Daniel W. C. Ho, Senior Member, IEEE
}

\begin{abstract}
In this paper, a new delay-dependent asymptotic stability condition for delayed Hopfield neural networks is given in terms of a linear matrix inequality, which is less conservative than existing ones in the literature. This condition guarantees the existence of a unique equilibrium point and its global asymptotic stability of a given delayed Hopfield neural network. Examples are provided to show the reduced conservatism of the proposed condition.
\end{abstract}

Index Terms-Global asymptotic stability, Hopfield neural networks, linear matrix inequality, time delays.

\section{INTRODUCTION}

$\mathbf{R}$ ECENTLY, there has been increasing interest in the study of Hopfield neural networks since Hopfield neural networks have found extensive applications in solving some optimization problems, associative memory, classification of patterns, reconstruction of moving images, and other areas [8]-[10]. It is now well known that applications of neural networks heavily depend on their dynamic behavior. Since stability is one of the most important issues related to such behavior, the problem of stability analysis of Hopfield neural networks has attracted considerable attention in recent years. Based on different assumptions on the network parameters, a great number of results on global asymptotic stability have been proposed; see, e.g., [1], [15], and the references therein.

In the implementation of artificial neural networks, however, time delays are unavoidable due to the finite switching speed of amplifiers. It has been shown that the existence of time delays in a neural network may lead to oscillation, divergence or instability. Therefore, the stability issue of Hopfield neural networks with time delays has been studied. Via different approaches, a great number of stability conditions for delayed Hopfield neural networks have been reported in the literature

Manuscript received March 9, 2005; revised June 13, 2005 and July 27, 2005. This work was supported in part by Hong Kong University under CRCG 10205878, the Program for New Century Excellent Talents in University by the Ministry of Education of China (NCET-04-0508), the National Natural Science Foundation of China under Grant 60304001, the Fok Ying Tung Education Foundation under Grant 91061, and the Foundation for the Author of National Excellent Doctoral Dissertation of China under Grant 200240. This paper was recommended by Associate Editor J. L. Moiola.

$\mathrm{S}$. Xu is with the Department of Automation, Nanjing University of Science and Technology, Nanjing 210094, China.

J. Lam is with the Department of Mechanical Engineering, University of Hong Kong, Hong Kong.

D. W. C. Ho is with the Department of Mathematics, City University of Hong Kong, Hong Kong.

Digital Object Identifier 10.1109/TCSII.2005.857764
[4]-[7], [12]-[14], [16], [20]. These stability results can be classified into two types; that is, delay-independent stability and delay-dependent stability; the former does not include any information on the size of delay while the latter employs such information. It is known that delay-dependent stability conditions are generally less conservative than delay-independent ones especially when the size of the delay is small. Although delay-dependent stability results for delayed Hopfield neural networks were proposed in [7], [18], [19], [21], and [22], they are sufficient conditions and have conservatism to some extent, which leaves open room for further improvement.

In this paper, we develop an improved delay-dependent asymptotic stability condition for Hopfield neural networks with time delays by utilizing Lyapunov functionals. Under the proposed condition, both the existence of a unique equilibrium point and the global asymptotic stability of a given delayed Hopfield neural network are guaranteed. The derived condition is expressed in terms of a linear matrix inequality (LMI), which can be checked numerically very efficiently by resorting to recently developed standard algorithms such as interior-point methods, and no tuning of parameters will be involved [3]. Examples are provided to demonstrate the reduced conservatism of the proposed condition.

Notation: Throughout this paper, for real symmetric matrices $X$ and $Y$, the notation $X \geq Y$ (respectively, $X>Y$ ) means that the matrix $X-Y$ is positive semi-definite (respectively, positive definite). The superscript " $T$ " represents the transpose. The notation $|\cdot|$ refers to the Euclidean vector norm. We use $\lambda_{\min }(\cdot)$ to denote the minimum eigenvalue of a real symmetric matrix. Matrices, if their dimensions are not explicitly stated, are assumed to have compatible dimensions.

\section{MAIn Results}

Consider a continuous time-delayed Hopfield neural network which is described by the following nonlinear retarded functional differential equations:

$\dot{x}_{i}(t)=-c_{i} x_{i}(t)+\sum_{j=1}^{n} a_{i j} g_{j}\left(x_{j}(t-\tau)\right)+b_{i}, \quad i=1,2, \ldots, n$

or equivalently

$$
\dot{x}(t)=-C x(t)+A g(x(t-\tau))+b
$$

where

$$
x(t)=\left[\begin{array}{llll}
x_{1}(t) & x_{2}(t) & \cdots & x_{n}(t)
\end{array}\right]^{T}
$$


is the state vector

$$
\begin{aligned}
& g(x(t-\tau)) \\
& \quad=\left[\begin{array}{lll}
g_{1}\left(x_{1}(t-\tau)\right) & g_{2}\left(x_{2}(t-\tau)\right) & \cdots g_{n}\left(x_{n}(t-\tau)\right)
\end{array}\right]^{T} .
\end{aligned}
$$

In (2), $C=\operatorname{diag}\left(c_{1}, c_{2}, \ldots, c_{n}\right)>0, A=\left\{a_{i j}\right\}$ are the delayed interconnection matrix representing the weighting coefficients of the neurons, $b=\left[\begin{array}{llll}b_{1} & b_{2} & \cdots & b_{n}\end{array}\right]^{T}$ is a constant input vector. The scalar $\tau>0$ is a constant delay of the system.

Throughout the paper, we make the following assumption.

Assumption 1: [2], [21]: The activation function $g(x)$ is boundless and satisfies

$$
0 \leq \frac{g_{i}\left(\xi_{1}\right)-g_{i}\left(\xi_{2}\right)}{\xi_{1}-\xi_{2}} \leq l_{i}, \quad i=1,2, \ldots, n
$$

for any $\xi_{1}, \xi_{2} \in \mathbb{R}$ and $\xi_{1} \neq \xi_{2}$.

Now, let $x^{*}=\left[\begin{array}{llll}x_{1}^{*} & x_{2}^{*} & \cdots & x_{n}^{*}\end{array}\right]^{T}$ be an equilibrium point of (2), and set

$$
y(t)=x(t)-x^{*}
$$

Then, it is easy to see that (2) can be transformed to

$$
\dot{y}(t)=-C y(t)+A f(y(t-\tau))
$$

where

$$
y(t)=\left[\begin{array}{llll}
y_{1}(t) & y_{2}(t) & \cdots & y_{n}(t)
\end{array}\right]^{T}
$$

is the state vector of the transformed system, and

$$
f(y(t))=\left[\begin{array}{llll}
f_{1}\left(y_{1}(t)\right) & f_{2}\left(y_{2}(t)\right) & \cdots & f_{n}\left(y_{n}(t)\right)
\end{array}\right]^{T}
$$

with

$$
f_{i}\left(y_{i}(t)\right)=g_{i}\left(y_{i}(t)+x_{i}^{*}\right)-g_{i}\left(x_{i}^{*}\right), \quad i=1,2, \ldots, n
$$

and $f_{i}(0)=0$, for $i=1,2, \ldots, n$. It is noted that $f_{i}(\cdot)$ satisfies (3); that is

$$
0 \leq \frac{f_{i}\left(\xi_{1}\right)-f_{i}\left(\xi_{2}\right)}{\xi_{1}-\xi_{2}} \leq l_{i}, \quad i=1,2, \ldots, n
$$

for any $\xi_{1}, \xi_{2} \in \mathbb{R}$ and $\xi_{1} \neq \xi_{2}$.

It can be seen that the global asymptotic stability of $x^{*}$ of (2) is equivalent to that of the trivial solution of (5). Therefore, in the next, attention will be focused on the study of the global asymptotic stability of (5).

Before presenting the main results, we give the following lemma, which will be used in the sequel.
Lemma 1: [17]: Let $\mathcal{D}, \mathcal{S}$ and $\mathcal{P}$ be real matrices of appropriate dimensions with $\mathcal{P}>0$. Then for any vectors $x, y$ with appropriate dimensions

$$
2 x^{T} \mathcal{D} \mathcal{S} y \leq x^{T} \mathcal{D} \mathcal{P} \mathcal{D}^{T} x+y^{T} \mathcal{S}^{T} \mathcal{P}^{-1} \mathcal{S} y
$$

Now, we are in a position to present a new asymptotic stability condition for system (5), which is dependent on the size of the delay.

Theorem 1: The origin of the delayed Hopfield neural network in (5) is the unique equilibrium point and it is globally asymptotically stable for any delay $0<\tau \leq \bar{\tau}$ if there exist matrices $P>0, Q>0, S>0, Y, Z$, and two diagonal matrices $W>0$ and $H>0$ such that the LMI shown in (7) at the bottom of the page, holds, where

$$
\begin{aligned}
L & =\operatorname{diag}\left(l_{1}, l_{2}, \ldots, l_{n}\right) \\
\Psi_{11} & =-P C-C P+Q-Y-Y^{T} \\
\Psi_{12} & =\frac{1}{2} P A L+Y-Z^{T} \\
\Psi_{22} & =Z+Z^{T}-Q+\frac{1}{4} L H L .
\end{aligned}
$$

Proof: We will first prove the uniqueness of the equilibrium point by contradiction. Let $\bar{y}$ be the equilibrium point of the delayed Hopfield neural network in (5). Then, we have

$$
-C \bar{y}+A f(\bar{y})=0
$$

Suppose $\bar{y} \neq 0$. Then, from (12), we have

$$
2 \bar{y}^{T} P[-C \bar{y}+A f(\bar{y})]=0
$$

which can be re-written as

$$
2 \bar{y}^{T} P\left(-C+\frac{1}{2} A L\right) \bar{y}+2 \bar{y}^{T} P A\left[\bar{F}(\bar{y})-\frac{1}{2} L\right] \bar{y}=0
$$

where

$$
\begin{aligned}
\bar{F}(y) & =\operatorname{diag}\left(\bar{F}_{1}\left(y_{1}\right), \bar{F}_{2}\left(y_{2}\right), \cdots \bar{F}_{n}\left(y_{n}\right)\right) \\
\bar{F}_{i}\left(y_{i}\right) & =\left\{\begin{array}{cl}
\frac{f_{i}\left(y_{i}\right)}{y_{i}}, & y_{i} \neq 0 \\
0, & y_{i}=0 .
\end{array}\right.
\end{aligned}
$$

By (6), we have that for $i=1,2, \ldots, n$

$$
-\frac{1}{2} l_{i} \leq \bar{F}_{i}\left(y_{i}\right)-\frac{1}{2} l_{i} \leq \frac{1}{2} l_{i}
$$

That is

$$
-\frac{1}{2} L \leq \bar{F}(\bar{y})-\frac{1}{2} L \leq \frac{1}{2} L
$$

$$
\left[\begin{array}{ccccc}
\Psi_{11}+\bar{\tau} C^{T} S C & \Psi_{12}-\frac{1}{2} \bar{\tau} C^{T} S A L & \bar{\tau} Y & \bar{\tau} C^{T} S A & P A \\
\Psi_{12}^{T}-\frac{1}{2} \bar{\tau} L A^{T} S C & \Psi_{22}+\frac{1}{4} \bar{\tau} L A^{T} S A L+\frac{1}{4} \bar{\tau} L W L & \bar{\tau} Z & -\frac{1}{2} \bar{\tau} L A^{T} S A & 0 \\
\bar{\tau} Y^{T} & \bar{\tau} Z^{T} & -\bar{\tau} S & 0 & 0 \\
\bar{\tau} A^{T} S C & -\frac{1}{2} \bar{\tau} A^{T} S A L & 0 & \bar{\tau} A^{T} S A-\bar{\tau} W & 0 \\
A^{T} P & 0 & 0 & 0 & -H
\end{array}\right]<0
$$


Therefore, using Lemma 1, we obtain

$$
\begin{aligned}
2 \bar{y}^{T} P A & {\left[\bar{F}(\bar{y})-\frac{1}{2} L\right] \bar{y} } \\
\leq & \bar{y}^{T} P A H^{-1} A^{T} P \bar{y}+\bar{y}^{T}\left[\bar{F}(\bar{y})-\frac{1}{2} L\right]^{T} \\
& \times H\left[\bar{F}(\bar{y})-\frac{1}{2} L\right] \bar{y} \\
\leq & \bar{y}^{T} P A H^{-1} A^{T} P \bar{y}+\frac{1}{4} \bar{y}^{T} L H L \bar{y} .
\end{aligned}
$$

This together with (14) gives

$$
\begin{aligned}
\bar{y}^{T}\left[P\left(-C+\frac{1}{2} A L\right)\right. & +\left(-C+\frac{1}{2} A L\right)^{T} P \\
& \left.+\frac{1}{4} L H L+P A H^{-1} A^{T} P\right] \bar{y} \geq 0 .
\end{aligned}
$$

On the other hand, considering (7), it can be shown that

$$
\left[\begin{array}{cc}
\Psi_{11}+P A H^{-1} A^{T} P & \Psi_{12} \\
\Psi_{12}^{T} & \Psi_{22}
\end{array}\right]<0
$$

That is, we get the inequality shown at the bottom of the page. Pre- and post-multiplying this inequality by

$$
\left[\begin{array}{ll}
I & I
\end{array}\right]
$$

and its transpose, respectively, we obtain

$$
\begin{aligned}
P\left(-C+\frac{1}{2} A L\right)+(-C+ & \left.\frac{1}{2} A L\right)^{T} P \\
& +\frac{1}{4} L H L+P A H^{-1} A^{T} P<0
\end{aligned}
$$

which contradicts with (18). Therefore, we have $\bar{y}=0$. That is, (5) has a unique equilibrium point.

Next, we show that the unique equilibrium point of (5) is globally asymptotically stable. To this end, we define a Lyapunov-Krasovskii functional candidate as

$$
V\left(y_{t}\right)=V_{1}\left(y_{t}\right)+V_{2}\left(y_{t}\right)+V_{3}\left(y_{t}\right)
$$

where

$$
y_{t}=y(t+\theta), \quad-2 \tau \leq \theta \leq 0
$$

and

$$
\begin{aligned}
& V_{1}\left(y_{t}\right)=y(t)^{T} P y(t) \\
& V_{2}\left(y_{t}\right)=\int_{t-\tau}^{t} y(\alpha)^{T} Q y(\alpha) d \alpha \\
& V_{3}\left(y_{t}\right)=\int_{-\tau}^{0} \int_{t+\beta}^{t} \dot{y}(\alpha)^{T} S \dot{y}(\alpha) d \alpha d \beta .
\end{aligned}
$$

Noting (15)-(17) and applying Lemma 1, we have that the timederivative of $V_{i}\left(x_{t}\right), i=1,2,3$, along the solution of (5) gives

$$
\begin{aligned}
\dot{V}_{1}\left(y_{t}\right)= & 2 y(t)^{T} P[-C y(t)+A \bar{F}(y(t-\tau)) y(t-\tau)] \\
\leq & 2 y(t)^{T} P\left[-C y(t)+\frac{1}{2} A L y(t-\tau)\right] \\
& +y(t)^{T} P A H^{-1} A^{T} P y(t) \\
& +y(t-\tau)^{T}\left[\bar{F}(y(t-\tau))-\frac{1}{2} L\right] \\
& \times H\left[\bar{F}(y(t-\tau))-\frac{1}{2} L\right] y(t-\tau) \\
\leq & 2 y(t)^{T} P\left[-C y(t)+\frac{1}{2} A L y(t-\tau)\right] \\
& +y(t)^{T} P A H^{-1} A^{T} P y(t) \\
& +\frac{1}{4} y(t-\tau)^{T} L H L y(t-\tau) \\
\dot{V}_{2}\left(y_{t}\right)= & y(t)^{T} Q y(t)-y(t-\tau)^{T} Q y(t-\tau) \\
\dot{V}_{3}\left(y_{t}\right)= & \int_{-\tau}^{0}\left[\dot{y}(t)^{T} S \dot{y}(t)-\dot{y}(t+\beta)^{T} S \dot{y}(t+\beta)\right] d \beta \\
= & \int_{t-\tau}^{t}\left[\dot{y}(t)^{T} S \dot{y}(t)-\dot{y}(\beta)^{T} S \dot{y}(\beta)\right] d \beta .
\end{aligned}
$$

Furthermore

$$
\begin{aligned}
\dot{y}(t)^{T} S \dot{y}(t) \leq & {\left[-C y(t)+\frac{1}{2} A L y(t-\tau)\right]^{T} } \\
& \times S\left[-C y(t)+\frac{1}{2} A L y(t-\tau)\right] \\
& +\left[-C y(t)+\frac{1}{2} A L y(t-\tau)\right]^{T} \\
& \times S A\left(W-A^{T} S A\right)^{-1} A^{T} S \\
& \times\left[-C y(t)+\frac{1}{2} A L y(t-\tau)\right] \\
& +\frac{1}{4} y(t-\tau)^{T} L W L y(t-\tau) .
\end{aligned}
$$

$$
\left[\begin{array}{cc}
-P C-C P+Q-Y-Y^{T}+P A H^{-1} A^{T} P & \frac{1}{2} P A L+Y-Z^{T} \\
\frac{1}{2} L A^{T} P+Y^{T}-Z & Z+Z^{T}-Q+\frac{1}{4} L H L
\end{array}\right]<0 .
$$


Now, by the Leibniz-Newton formula, it is easy to show that

$$
y(t-\tau)=y(t)-\int_{t-\tau}^{t} \dot{y}(\alpha) d \alpha .
$$

This together with (22)-(25) gives

$$
\begin{aligned}
& \dot{V}\left(y_{t}\right) \leq 2 y(t)^{T} P\left[-C y(t)+\frac{1}{2} A L y(t-\tau)\right] \\
& +y(t)^{T} P A H^{-1} A^{T} P y(t) \\
& +\frac{1}{4} y(t-\tau)^{T} L H L y(t-\tau)+y(t)^{T} Q y(t) \\
& -y(t-\tau)^{T} Q y(t-\tau) \\
& +\int_{t-\tau}^{t}\left\{\left[-C y(t)+\frac{1}{2} A L y(t-\tau)\right]^{T}\right. \\
& \times S\left[-C y(t)+\frac{1}{2} A L y(t-\tau)\right] \\
& +\left[-C y(t)+\frac{1}{2} A L y(t-\tau)\right]^{T} \\
& \times S A\left(W-A^{T} S A\right)^{-1} A^{T} S \\
& \times\left[-C y(t)+\frac{1}{2} A L y(t-\tau)\right]+\frac{1}{4} y(t-\tau)^{T} \\
& \left.\times L W L y(t-\tau)-\dot{y}(\beta)^{T} S \dot{y}(\beta)\right\} d \beta \\
& +2 y(t)^{T} Y \int_{t-\tau}^{t} \dot{y}(\alpha) d \alpha-2 y(t)^{T} Y[y(t)-y(t-\tau)] \\
& +2 y(t-\tau)^{T} Z \int_{t-\tau}^{t} \dot{y}(\alpha) d \alpha-2 y(t-\tau)^{T} \\
& \times Z[y(t)-y(t-\tau)] \\
& =\frac{1}{\tau} \int_{t-\tau}^{t} \delta(t, \beta)^{T} \Pi(\tau) \delta(t, \beta) d \beta
\end{aligned}
$$

where

$$
\begin{aligned}
\Pi(\tau)= & {\left[\begin{array}{ccc}
\Psi_{11}+P A H^{-1} A^{T} P & \Psi_{12} & \tau Y \\
\Psi_{12}^{T} & \Psi_{22}+\frac{1}{4} \tau L W L & \tau Z \\
\tau Y^{T} & \tau Z^{T} & -\tau S
\end{array}\right] } \\
& +\left[\begin{array}{c}
C^{T} \\
-\frac{1}{2} L A^{T} \\
0
\end{array}\right]\left[\tau S+\tau S A\left(W-A^{T} S A\right)^{-1} A^{T} S\right] \\
& \times\left[\begin{array}{c}
C^{T} \\
-\frac{1}{2} L A^{T} \\
0
\end{array}\right] \\
\delta(t, \beta)= & {\left[\begin{array}{lll}
y(t)^{T} & y(t-\tau)^{T} & \dot{y}(\beta)^{T}
\end{array}\right]^{T} }
\end{aligned}
$$

where $\Psi_{11}, \Psi_{12}$ and $\Psi_{22}$ are given in (9)-(11), respectively. Now, applying the Schur complement formula to the LMI in (7) results in

$$
\begin{aligned}
& {\left[\begin{array}{cc}
\Psi_{11}+P A H^{-1} A^{T} P & \Psi_{12} \\
\Psi_{12}^{T} & \Psi_{22}+\frac{1}{4} \bar{\tau} L W L
\end{array}\right]+\left[\begin{array}{c}
-C^{T} \\
\frac{1}{2} L A^{T}
\end{array}\right]} \\
& \quad \times\left[\bar{\tau} S+\bar{\tau} S A\left(W-A^{T} S A\right)^{-1} A^{T} S\right]\left[\begin{array}{c}
-C^{T} \\
\frac{1}{2} L A^{T}
\end{array}\right]^{T} \\
& \quad+\left[\begin{array}{c}
Y \\
Z
\end{array}\right] \bar{\tau} S^{-1}\left[\begin{array}{l}
Y \\
Z
\end{array}\right]^{T}<0 .
\end{aligned}
$$

Then, it is easy to show that for all $0<\tau \leq \bar{\tau}$, we have

$$
\begin{aligned}
& {\left[\begin{array}{cc}
\Psi_{11}+P A H^{-1} A^{T} P & \Psi_{12} \\
\Psi_{12}^{T} & \Psi_{22}+\frac{1}{4} \tau L W L
\end{array}\right]} \\
& +\left[\begin{array}{c}
C^{T} \\
-\frac{1}{2} L A^{T}
\end{array}\right]\left[\tau S+\tau S A\left(W-A^{T} S A\right)^{-1} A^{T} S\right] \\
& \quad \times\left[\begin{array}{c}
C^{T} \\
-\frac{1}{2} L A^{T}
\end{array}\right]^{T}+\left[\begin{array}{c}
Y \\
Z
\end{array}\right] \tau S^{-1}\left[\begin{array}{c}
Y \\
Z
\end{array}\right]^{T}<0
\end{aligned}
$$

which, by the Schur complement formula, implies $\Pi(\tau)<0$. This together with (26) gives

$$
\dot{V}\left(y_{t}\right) \leq-k\|y(t)\|^{2}
$$

where $k=(1 / \tau) \lambda_{\min }(-\Upsilon(\tau))>0$. Finally, by [11], it follows from (27) that the delayed Hopfield neural network in (5) is asymptotically stable for any delay $0<\tau \leq \bar{\tau}$. This completes the proof.

Remark 1: Theorem 1 provides a new delay-dependent global asymptotic stability condition for delayed Hopfield neural networks in terms of an LMI; this is derived by choosing an appropriate Lyapunov functional different from those in [7], [19], [21], which leads to a less conservative stability condition in (7).

\section{EXAMPLES}

In this section, we provide examples to illustrate the reduced conservatism of Theorem 1 by comparing it with recently reported delay-dependent asymptotic stability results in the literature.

Example 1: [21]: Consider a delayed Hopfield network described by

$$
\begin{aligned}
& \dot{x}_{1}(t)=-0.7 x_{1}(t)+0.1 g_{1}\left(x_{1}(t-\tau)\right)+0.1 g_{2}\left(x_{2}(t-\tau)\right)-2 \\
& \dot{x}_{2}(t)=-0.7 x_{1}(t)+0.3 g_{1}\left(x_{1}(t-\tau)\right)+0.3 g_{2}\left(x_{2}(t-\tau)\right)+1
\end{aligned}
$$

where the activation function is given as

$$
g_{1}(x)=g_{2}(x)=0.5(|x+1|-|x-1|)
$$


Then, it is easy to see that

$$
C=\left[\begin{array}{cc}
0.7 & 0 \\
0 & 0.7
\end{array}\right] \quad A=\left[\begin{array}{ll}
0.1 & 0.1 \\
0.3 & 0.3
\end{array}\right] \quad L=\left[\begin{array}{ll}
1 & 0 \\
0 & 1
\end{array}\right]
$$

By the methods in [7], [19] and [21], the maximum delay $\bar{\tau}$ that guarantees the delayed Hopfield network to be asymptotically stable are calculated to be $0.3636,2.2361$ and 0.4926 , respectively. However, by Theorem 1 in this paper, it is found that LMI (7) is feasible for any arbitrarily large $\bar{\tau}$ (as long as the numerical computation is reliable). Therefore, it can be seen that Theorem 1 provides a less conservative condition for this example.

Example 2: Consider a delayed Hopfield network in (5) with parameters

$$
\begin{aligned}
C & =\left[\begin{array}{ccc}
4.1989 & 0 & 0 \\
0 & 0.7160 & 0 \\
0 & 0 & 1.9985
\end{array}\right] \\
A & =\left[\begin{array}{ccc}
-0.1052 & -0.5069 & -0.1121 \\
-0.0257 & -0.2808 & 0.0212 \\
0.1205 & -0.2153 & 0.1315
\end{array}\right] \\
L & =\left[\begin{array}{ccc}
0.4219 & 0 & 0 \\
0 & 3.8993 & 0 \\
0 & 0 & 1.0160
\end{array}\right] .
\end{aligned}
$$

For this example, both of the delay-dependent conditions in [7], [21], [22] cannot be satisfied for any $\tau>0$. Therefore, they cannot provide any results on the maximum allowed delay $\bar{\tau}$. However, by the method in [18] and [19], we obtain $\bar{\tau}$ as 1.7484 and 0.4121 , respectively, while by Theorem 1 in this paper, we have $\bar{\tau}=1.7644$, which also shows that the condition given in Theorem 1 is less conservative than those in [7], [18], [19], [21], and [22].

\section{CONCLUSION}

An improved delay-dependent asymptotic stability condition for Hopfield neural networks with time delays has been developed in this paper. This condition is expressed in terms of an LMI, which can be checked easily. Examples have been provided to demonstrate the less conservatism of the proposed results.

\section{REFERENCES}

[1] S. Arik, "Global asymptotic stability of a class of dynamical neural networks," IEEE Trans. Circuits Syst. I, Fundam. Theory Appl., vol. 47, no. 4, pp. 568-571, Apr. 2000
[2] - "Global asymptotic stability of a larger class of neural networks with constant time delay," Phys. Lett. A, vol. 311, pp. 504-511, 2003.

[3] S. Boyd, L. El Ghaoui, E. Feron, and V. Balakrishnan, Linear Matrix Inequalities in System and Control Theory. Philadelphia, PA: SIAM, 1994.

[4] J. Cao, "Global stability conditions for delayed CNNs," IEEE Trans. Circuits Syst. I, Fundam. Theory Appl., vol. 48, no. 11, pp. 1330-1333, Nov. 2001.

[5] J. Cao and D. W. C. Ho, "A general framework for global asymptotic stability analysis of delayed neural networks based on LMI approach," Chaos, Solitons, Fractals, vol. 24, pp. 1317-1329, 2005.

[6] J. Cao and J. Wang, "Global asymptotic and robust stability of recurrent neural networks with time delays," IEEE Trans. Circuits Syst. I, Fundam. Theory Appl., vol. 52, no. 2, pp. 417-426, Feb. 2005.

[7] A. Chen, J. Cao, and L. Huang, "An estimation of upperbound of delays for global asymptotic stability of delayed Hopfield neural networks," IEEE Trans. Circuits Syst. I, Fundam. Theory Appl., vol. 49, no. 7, pp. 1028-1032, Jul. 2002.

[8] A. Cichocki and R. Unbehauen, Neural Networks for Optimization and Signal Processing. Chichester, U.K.: Wiley, 1993.

[9] J. Farrell and A. Michel, "A synthesis procedure for Hopfield's continuous-time associative memory," IEEE Trans. Circuits Syst., vol. 37, no. 7, pp. 877-884, Jul. 1990.

[10] J. J. Hopfield, "Neurons with graded response have collective computational properties like those of two-state neurons," Proc. Natl. Acad. Sci. USA, vol. 81, pp. 3088-3092, 1984.

[11] V. B. Kolmanovskii and A. D. Myshkis, Introduction to the Theory and Applications of Functional Differential Equations. Dordrecht, The Netherlands: Kluwer Academic, 1999.

[12] X. Liao, G. Chen, and E. N. Sanchez, "Delay-dependent exponential stability analysis of delayed neural networks: an LMI approach," Neural Netw., vol. 15, pp. 855-866, 2002.

[13] — - "LMI-based approach for asymptotically stability analysis of delayed neural networks," IEEE Trans. Circuits Syst. I, Fundam. Theory Appl., vol. 49, no. 7, pp. 1033-1039, Jul. 2002.

[14] — "Delay-dependent exponential stability analysis of delayed neural networks: an LMI approach," Neur. Networks, vol. 16, pp. 1401-1402, 2003.

[15] A. N. Michel, J. A. Farrel, and W. Porod, "Qualitative analysis of neural networks," IEEE Trans. Circuits Syst., vol. 36, no. 2, pp. 229-243, Feb. 1989.

[16] B. Xu, X. Liu, and X. Liao, "Global asymptotic stability of high-order Hopfield type neural networks with time delays," Comput. Math. Appl., vol. 45, pp. 1729-1737, 2003.

[17] S. Xu, T. Chen, and J. Lam, "Robust $H_{\infty}$ filtering for uncertain Markovian jump systems with mode-dependent time delays," IEEE Trans. Autom. Contr., vol. 48, no. 5, pp. 900-907, May 2003.

[18] S. Xu, J. Lam, D. W. C. Ho, and Y. Zou, "Novel global asymptotic stability criteria for delayed cellular neural networks," IEEE Trans. Circuits Syst. II, Analog Digit. Signal Process., vol. 52, no. 6, pp. 349-353, Jun. 2005.

[19] H. Ye, A. N. Michel, and K. Wang, "Global stability and local stability of Hopfield neural networks with delays," Phys. Rev. E, vol. 50, pp. 4206-4213, 1994.

[20] E. Yucel and S. Arik, "New exponential stability results for delayed neural networks with time varying delays," Physica D, vol. 191, pp. 314-322, 2004.

[21] Q. Zhang, X. Wei, and J. Xu, "Global asymptotic stability of Hopfield neural networks with transmission delays," Phys. Lett. A, vol. 318, pp. 399-405, 2003.

[22] Q. Zhang, X. Wei, and J. Xu, "Delay-dependent exponential stability of cellular neural networks with time-varying delays," Chaos, Solitons, Fractals, vol. 23, pp. 1363-1369, 2005. 